Revisão / Review

\title{
Políticas públicas para o controle da anemia ferropriva
}

\section{Public policies to control iron deficiency in Brazil}

Sophia C. Szarfarc

\begin{abstract}
A anemia por deficiência de ferro configura um problema epidemiológico da maior relevância atuando nos gastos públicos de saúde, nas consequências sociais do aumento de riscos no período gestacional, na redução da produtividade e, ainda, nas consequências, em longo prazo, do desenvolvimento mental. Algumas datas marcamo envolvimento do governo brasileiro em busca de alternativas de controle da deficiência marcial: 1977 - $1^{a}$ Reunião do Ministério da Saúde (INAN), com especialistas nacionais e internacionais, para discutir perspectivas e intervenções para o controle da anemia; 1982/83 - distribuição de suplemento de ferro para a clientela do Programa de Atenção à Gestante e dosagem de hemoglobina na $1^{a}$ consulta; 1992 - assinatura de compromisso brasileiro de reduzir em 1/3 a prevalência de anemia em gestantes; 1994 - implantação do Programa de Leite Vivaleite, no estado de São Paulo, fornecendo leite fortificado com ferro a famílias com crianças até 6 anos e renda inferior a dois salários mínimos; 2002/junho 2004 - fortificação das farinhas de trigo e de milho com ferro; 2005 - programa de suplementação de ferro a lactentes; 2009/março-divulgação do resultado do levantamento de prevalência de anemia em mulheres (15-49 anos) e crianças (6 - 59 meses) no Brasil; 2009/agosto - foi reeditada a Portaria no 1793/GM/ agosto/2009 do Ministério da Saúde, instituindo a Comissão Interinstitucional para implementação, acompanhamento e monitorização das ações de fortificação das farinhas de trigo e milho e seus subprodutos. Rev. Bras. Hematol. Hemoter. 2010; 32 (Supl.2):2-7.
\end{abstract}

Palavras-chave: Políticas públicas; deficiência de ferro; nutrição em Saúde Pública.

\section{Introdução}

Em 1992, o Brasil assumiu um compromisso junto às Nações Unidas de, até o ano 2000 (prorrogado para 2003), reduzir a prevalência de anemia por deficiência de ferro entre gestantes (posteriormente expandido para crianças em idade pré-escolar) em 1/3 dos níveis encontrados em 1990. ${ }^{1}$ Embora pouco ambicioso na proposta de controle da anemia, o compromisso brasileiro teve o mérito de intensificar os estudos de intervenção para controlar a deficiência do ferro e erradicar a sua forma mais grave: a anemia. Sem exceção, todos os projetos desenvolvidos desde então, tinham uma meta comum: a busca de propostas de intervenção factíveis de im- plantação visando o controle da desnutrição em populações de risco.

As perdas econômicas decorrentes da anemia não podem ser desprezadas. Relatório do Banco Mundial, de 1994, ${ }^{2}$ destacava que, apesar da dificuldade em se quantificar o custo para o Brasil, cerca de 5\% do PIB de países em desenvolvimento são desperdiçados com os gastos em saúde decorrentes da anemia por deficiência de ferro. Transpondo esses cálculos para o ano de 2008, pode-se dizer que, o Brasil, com um PIB estimado em R \$ 2,3 trilhões, gastou, neste ano, R \$ 116 bilhões para tratar problemas de saúde decorrentes da deficiência de ferro. Horton e Ross ${ }^{3}$ ressaltam o elevado custo indireto da deficiência de ferro na redução da produtivida-

Deficiencias Nutricionais - Professor aposentado.

Faculdade de Saúde Pública, Universidade de São Paulo (USP) - São Paulo-SP.

Correspondência: Sophia Cornbluth Szarfarc

Av. Dr. Arnaldo, 715

01246-904 - São Paulo-SP - Brasil

Tel./Fax.: (55 11) 3061-7705; (55 11) 3061-7771

E-mail:scfarc@usp.br

Doi: 10.1590/S1516-84842010005000065 
de, na mortalidade materna, na prematuridade, no baixo peso das crianças ao nascimento e, principalmente, nas conseqüências irreversíveis sobre o desenvolvimento cognitivo que ocorrem na infância e se traduzem no decorrer da vida em reprovação e abandono escolar, acompanhados de baixa capacidade física para trabalhos braçais. Eles afirmam que tanto a fortificação de alimentos habituais na alimentação da população alvo, como a suplementação medicamentosa, se efetivamente implantadas, constituem um investimento pequeno, inferior a $0,3 \%$ do PIB de países em desenvolvimento.

O ano de 1977 representou um marco no controle da anemia nutricional no Brasil. Nesse ano, o extinto Instituto Nacional de Alimentação e Nutrição (INAN), do Ministério da Saúde, organizou uma reunião técnica para discutir o problema da deficiência de ferro no País. ${ }^{4}$ Os estudos de diagnóstico até então desenvolvidos eram poucos e pontuais, porém permitiam concluir que a anemia ocorria em proporção endêmica. Como continua sendo aceito, a patologia foi atribuída à deficiência alimentar de ferro e, assim sendo, as propostas de intervenção que vinham sendo adotadas em todo o mundo, tinham como objetivo, o aumento do consumo do mineral.

Além de destacarem que o consumo deficiente e/ou inadequado do mineral era o seu principal determinante, os especialistas, nacionais e internacionais, reforçaram que as conseqüências ocasionadas por essa desnutrição específica eram deletérias para a qualidade de vida da população, em especial das gestantes e seus conceptos. ${ }^{5}$

Demorou, porém em 1982/83, foi implantada, no País, a distribuição de ferro suplementar para a totalidade da clientela atendida pelo Programa de Atenção à Gestante (PAG) das Unidades Básicas de Saúde (UBS). Conjuntamente ao suplemento, o diagnóstico de anemia, através da dosagem da concentração de hemoglobina, deveria ser realizado por ocasião da inscrição da mulher no Programa (MS). ${ }^{6}$ Diferente do que ocorre atualmente, eram raras as UBSs que colhiam o material necessário para o diagnóstico de anemia.

Um levantamento de prevalência de anemia entre gestantes atendidas em serviços públicos de saúde do estado de São Paulo, que mantinham a dosagem da concentração de hemoglobina na rotina do PAG, foi realizado três anos após a implantação da distribuição do suplemento marcial. ${ }^{7}$ Neste estudo foi verificado que $35,1 \%$ das gestantes ingressantes no PAG apresentavam concentração de hemoglobina ([Hb]) inferior a 11,0 g/dL, valor que, de acordo com a Organização Mundial de Saúde, ${ }^{8}$ caracterizava a presença de anemia. Embora elevada, a prevalência de anemia encontrada estava bastante subestimada, como já havia sido ressaltado no estudo, uma vez que durante uma gestação normal, os valores hematológicos de hemoglobina mudam substancialmente. Para mulheres com nutrição de ferro adequada, o que é desejável, a $[\mathrm{Hb}]$ inicia o declínio no final do $1^{\circ}$ trimestre de gestação e atinge o valor mínimo no final do $2^{\circ}$ trimestre quando, gradual e lentamente aumenta até o final do processo. ${ }^{9}$
Vale a pena, no entanto, ressaltar que, mesmo adotando o valor único de referência proposta pela OMS, a prevalência encontrada $(35,1 \%)$ já seria suficientemente elevada para indicar que os programas de intervenção deveriam ser oferecidos não somente para as gestantes, mas, também para outros grupos populacionais. Mulheres anêmicas são gestantes anêmicas e geram filhos também anêmicos que, ingerindo alimentação deficiente no mineral também se mantêm anêmicos, com risco de sofrer todos os agravos decorrentes da desnutrição. ${ }^{10}$

$\mathrm{O}$ aumento da incidência de prematuridade e/ou peso baixo ao nascer nos conceptos, que incide com maior freqüência entre gestantes anêmicas, afeta de forma decisiva o desenvolvimento da criança e o risco dela se tornar anêmica já ao nascimento. Por sua vez, crianças anêmicas têm sido consideradas as mais sensíveis a agravos no desenvolvimento cognitivo e neuropsicomotor em função dos comprometimentos de processos fisiológicos relacionados à produção de hemoglobina e consequente transporte de oxigênio ao cérebro, alterando a neurotransmissão e mielinização. ${ }^{11}$

Devido a dificuldades decorrentes dos inúmeros fatores sociais, ambientais e econômicos que concorrem para o desenvolvimento de uma criança, poucos são os estudos que avaliam a inter-relação entre deficiência de ferro e desenvolvimento cognitivo. No entanto, como refere GranthamMcGregor, ${ }^{12}$ a possibilidade que a deficiência de ferro afete $o$ desenvolvimento infantil é um sério problema de saúde pública e traz implicações não somente para o desenvolvimento pessoal, mas também para as nações em desenvolvimento onde a prevalência da anemia é sempre elevada. ${ }^{10}$

Embora tenha uma inconteste importância epidemiológica e acarrete elevados custos públicos e privados de saúde, é muito recente o interesse (possivelmente por não apresentar sinais e/ou sintomas específicos) dos governos pelo problema das anemias como tema de políticas públicas. Foi a partir da Reunião de Cúpula de New York, promovida pelas Nações Unidas, que a anemia passou a figurar no elenco das prioridades mundiais de saúde e nutrição. ${ }^{13} \mathrm{O}$ compromisso assinado pelo Brasil, em 1992, de reduzir em 1/3 a prevalência de anemia entre gestantes até o ano 2000, postergado para 2003 e ampliado para crianças em idade préescolar, embora pouco ambicioso nas suas metas, teve o mérito de intensificar os estudos de diagnóstico e intervenção para controlar a deficiência do ferro e erradicar a sua forma mais grave: a anemia.

A identificação da anemia como um dos fatores causais de inadequado desenvolvimento cognitivo, social, motor, e de redução da capacidade de aprendizagem entre crianças, colocou-as, ao lado das gestantes, como grupo prioritário dentro dos programas de intervenção nutricional de controle da deficiência de ferro. ${ }^{14}$

As prevalências de anemia entre os indivíduos desse grupo populacional são extremamente elevadas, como mostraram Fisberg et al..${ }^{15}$ que registraram $75 \%$ de anemia em 
crianças de creches brasileiras, Torres et al., ${ }^{16}$ que encontraram 59\% de anêmicos entre crianças paulistas de 6 a 23 meses de idade, atendidas nas Unidades Básicas de Saúde e Szarfarc et al.,${ }^{17}$ que encontraram $56 \%$ de crianças que frequentam serviços públicos de saúde do Brasil, no primeiro ano de vida, com anemia.

Conscientes da importância do ferro na alimentação infantil e do leite na prática alimentar da criança, Torres e Queiroz ${ }^{18}$ lideraram uma série de experimentos com leite fluido fortificado com ferro proveniente de diferentes fontes. Concluíram desses estudos que a eficácia do suplemento no controle da deficiência do ferro era maior quando o composto utilizado para a fortificação era o ferro bisglicina quelato (Ferrochel ${ }^{\circledR}$ ). Ao iniciar o estudo de intervenção, 74,4\% das 270 crianças do município de Angatuba, frequentadoras da UBS (Unidade Básica de Saúde) que participaram do projeto estavam anêmicas. Ao final de cinco meses, o porcentual de anemia baixou para $45,6 \%$, e, ao final de 13 meses, apenas $4,1 \%$ das crianças apresentavam um diagnóstico positivo para anemia.

Aliando esses conhecimentos à vontade política, já em 1997 a Secretaria de Agricultura do Estado de São Paulo implantou o programa do leite Vivaleite, que veio a ser normatizado em $1999 .{ }^{19}$ Por este programa, famílias com renda inferior a dois salários mínimos e filhos com idade entre 6 e 72 meses recebiam o Vivaleite em quantidade equivalente a meio litro diário. A efetividade do Vivaleite, cuja eficácia era tão elevada, não foi a esperada. A diluição intrafamiliar do alimento, a embalagem plástica, sem as características de "longa vida", e a ausência de um programa de educação alimentar permanente acompanhando a distribuição do produto fortificado foram as principais causas detectadas para justificar a baixa efetividade encontrada. ${ }^{20,21}$

A grande demonstração da sintonia do governo brasileiro com as recomendações internacionais e da vontade política de minimizar a anemia dentre os problemas de saúde pública da população brasileira foi o Programa de Fortificação de Farinhas de Trigo e de Milho, para todos os fins, com ferro e ácido fólico, cuja efetiva implantação ocorreu em junho de 2004 (http://www.anvisa.gov.br/legis/resol/2002/ 344_02rdc.htm.)

Ainda visando cumprir o compromisso firmado e conscientes do baixo consumo de alimentos sólidos por lactentes, em maio de 2005 foi editado, pelo Ministério de Saúde, o Programa Nacional de Suplemento de Ferro (PNSFe), a ser distribuído através das UBSs a crianças de 6 a 18 meses de idade (http://www.saude.gov.br/nutricao).

São poucos e contraditórios os resultados de estudos de avaliação da efetividade desses dois programas de intervenção.

Um estudo de abrangência nacional, coordenado por Fujimori e Szarfarc, ${ }^{22}$ com o objetivo de avaliar o impacto da fortificação das farinhas fortificadas na ocorrência da anemia em gestantes, permitiu ver que, na maior parte dos locais estudados, a prevalência de anemia após, pelo menos, um ano da implantação da intervenção, era elevada e similar àquela encontrada antes da fortificação. A média de prevalência de anemia em 2002, 22,4\%, não diferia estatisticamente daquela encontrada após julho de 2005: 20,4\%. ${ }^{22}$

Dos resultados encontrados em municípios de todas as regiões brasileiras, duas hipóteses foram levantadas. A primeira refere-se ao baixo consumo dos alimentos fortificados. Colaborando com essa hipótese verifica-se esse baixo consumo nos dados levantados pelo IBGE (2004) na Pesquisa de Orçamentos Familiares POF (2002) (http//ftp.ibge.br/ orçamentos familiares/pesquisa de orçamentos familiares 2002 2003/analise)

Como mostra o Quadro 1, especialmente entre a população de mais baixa renda ( $<2$ salários mínimos/familia), a quantidade de ferro suplementar é insuficiente para suprir ao menos os $30 \%$ ( $4,2 \mathrm{mg} \mathrm{Fe} / \mathrm{dia})$ do nutriente propostos para identificar alimentos fortificados.

Quadro 1. Consumo de derivados de farinha de trigo (pão, massas e bolachas) g/dia, para todas as classes de renda e para $<2$ SM. POF $2002 / 3$

\begin{tabular}{ccccc}
\hline Região & Qtd g/dia & $<2$ S.M. & {$[\mathrm{Fe}] \mathrm{mg}$} & $\begin{array}{c}\text { [Fe] mg } \\
<2 \text { S.M. }\end{array}$ \\
\hline Brasil & 66,8 & 16,2 & 2,27 & 0,54 \\
Norte & 51,0 & 13,2 & 1,73 & 0,35 \\
Nordeste & 66,0 & 16,3 & 2,24 & 0,55 \\
Sudeste & 72,3 & 18,2 & 2,46 & 0,61 \\
Sul & 70,4 & 17,4 & 2,39 & 0,60 \\
Centro-Oeste & 45,5 & 11,4 & 1,55 & 0,39 \\
\hline
\end{tabular}

Fonte: IBGE, POF, 2004

O segundo aspecto discutido com vistas a entender os resultados encontrados na avaliação da efetividade das farinhas diz respeito à possibilidade de melhorar o aproveitamento do mineral utilizando compostos de biodisponibilidade elevada e cuja absorção sofra baixa influência de inibidores de seu aproveitamento.

O Quadro 2 apresenta a absorção potencial do ferro suplementar veiculado pela farinha de trigo utilizando o sulfato ferroso ou o Ferrochel ${ }^{\circledR}$ como fonte do mineral, utilizando como modelo diferentes alimentos que estão disponiveis no mercado varejista na forma de fortificados e que fazem parte da prática alimentar nacional.

Quadro 2. Absorção potencial do ferro suplementar de alimentos fortificados com ferro veiculado por diferentes compostos

\begin{tabular}{ccccc}
\hline \multicolumn{4}{c}{ fortificados com ferro veiculado por diferentes compostos } \\
\hline Alimento & arroz & leite & macarrão & pão \\
\hline Quantidade $(\mathrm{g})$ & 50 & 200 & 100 & 50 \\
$\begin{array}{c}\text { [Fe] suplementar }(\mathrm{mg}) \\
\text { 3\% Fe absorvível do } \\
\quad \text { FeSO }\end{array}$ & 0,065 & 4,2 & 3,36 & 1,7 \\
$\begin{array}{c}16 \% \mathrm{Fe}_{4} \text { absorvivel do } \\
\text { Fechel® }\end{array}$ & 0,345 & 0,690 & 0,010 & 0,050 \\
\end{tabular}


Os dados do Quadro 2 permitem ressaltar que há uma grande diferença na perspectiva de sucesso do programa decorrente da escolha do composto fonte de ferro.

Dentre os poucos trabalhos divulgados no Brasil após a fortificação das farinhas, Assunção et al. ${ }^{23}$ em estudo de série temporal com amostra probabilística da população de crianças de 0 a 5 anos de idade, da cidade de Pelotas (Rio Grande do Sul), analisaram a prevalência de anemia domiciliar nos anos de 2004 (antes da fortificação), 2005 e 2006 (12 e 24 meses após a fortificação) e não observaram qualquer efeito significativo nos níveis médios de hemoglobina dos pré-escolares.

No entanto, a efetividade das farinhas fortificadas no controle da anemia pode ser comprovada em grupos populacionais onde o consumo dos derivados do trigo é frequente e a quantidade com que é consumida é regular. Queiroz et $a l .{ }^{24}$ verificaram entre escolares ingressantes de um município paulista, cuja merenda escolar compreendia o almoço e um lanche, a diminuição na prevalência de anemia de $30 \%$ (valor médio de prevalência encontrado na região) para 5,8\%, sendo que houve um aumento de $22,5 \%$ deferro biodisponivel. Também Costa et al..$^{25}$ encontraram resultado similar entre crianças de 2 a 5 anos que frequentavam creches assistenciais da regional Butantã, São Paulo. De 64\% de anêmicos descritos para a população de creches da região em 2002, este estudo descreve a anemia em $21 \%$ dos pré-escolares.

Embora não se possa fazer uma associação de causa e efeito sobre a efetividade e eficácia da fortificação das farinhas com ferro sobre a acentuada redução observada na proporção de anemia na região Butantã da cidade de São Paulo, uma vez que o presente estudo apenas analisou a adequação programada das refeições presentes nos cardápios dos pré-escolares, os resultados encontrados por Queiroz et al. ${ }^{24}$ e Costa et al. ${ }^{25}$ são estimuladores e importantes para os gestores públicos.

A eficácia de uma suplementação semanal com sulfato ferroso - que serviu de modelo para o PNSFe - foi avaliada no município de São Paulo, ${ }^{26} \mathrm{em}$ uma coorte de 1.015 crianças de 4 a 59 meses de idade divididas em dois grupos: controle (GC) e intervenção (GI). No GC após a avaliação da [Hb], a mãe era informada do resultado e, no caso de diagnóstico positivo de anemia, era orientada a procurar um serviço de saúde para tratamento. As crianças do GI receberam, independente do resultado de anemia, solução de sulfato ferroso com orientação para ingestão de ao redor de $4 \mathrm{mgFe} / \mathrm{kg}$ de peso, uma vez por semana. Alguns resultados merecem ser destacados:

- após período de aproximadamente 7 meses, a [Hb] média do GI foi significativamente maior do que do GC;

- a incidência de anemia em crianças a partir dos 14 meses de idade foi significativamente menor entre as do GI do que nas do GC;

- a adesão plena e parcial somou $62 \%$ das crianças. $\mathrm{O}$ efeito benéfico da suplementação foi relatado pelas mães com frequência duas vezes maior do que de efeitos negativos. Quanto a esses, eles não estavam relacionados à diminuição da adesão.

A comprovação que a suplementação semanal foi eficaz para prevenir, evitando o declínio e estabilizando os níveis de hemoglobina, embora não associada à elevação desse indicador, permitiram propô-la como uma alternativa viável no controle da anemia. ${ }^{26}$ Dessa forma, o modelo paulistano foi adotado como estratégia no "Projeto para controle da anemia ferropriva em crianças menores de dois anos", nos 512 municípios nordestinos incluídos no Programa de Redução da Mortalidade Infantil ${ }^{27} \mathrm{e}$, como já descrito, desde maio de 2005, foi estendido para todo o Brasil. ${ }^{28}$

A efetividade dos programas de suplementação esbarra em uma série de fatores relevantes, políticos, administrativos, econômicos, de planejamento e gestão, de organização, fatores materiais e humanos, que envolvem, em última análise, interesses, motivações e crenças. Há ainda que se considerar que o sulfato ferroso, escolhido como suplemento pelo seu baixo custo e razoável biodisponibilidade, provoca efeitos colaterais indesejáveis como náuseas, cólicas abdominais, obstipação e/ou diarreia, e sabor metálico característico (como a maioria dos sais de ferro) o que faz com que a rejeição à sua ingestão seja frequente.

Além desses aspectos, contribuem para a baixa adesão o fato de que, para controlar a deficiência, a suplementação medicamentosa deva ser utilizada por tempo prolongado e de que pessoas não associam com a anemia quaisquer sinais e/ou sintomas específicos, e, portanto não associam com a suplementação a melhora do quadro clínico. Na suplementação, o ferro é ingerido como medicamento para uma "doença" sem uma sintomatologia que justifique seu uso. Nesse sentido, a ingestão de alimentos fortificados parece ser a solução definitiva e mais adequada para atender à demanda da população brasileira

O controle da deficiência de ferro mostra que, se por um lado, aparentemente a anemia é uma patologia de fácil solução - o simples aumento da ingestão de ferro modificaria a situação epidemiológica da deficiência, por outro lado, o modelo adotado para programas implantados em serviços de saúde, incluindo o Programa de Atenção à Gestante do Ministério da Saúde, ressalta a existência de problemas operacionais que impedem o surgimento do efeito esperado.

Entre os problemas detectados, é nítido que o sulfato ferroso, composto de opção nos vários programas nacionais, apresenta várias restrições que diminuem a adesão ao seu consumo: o sabor desagradável, a necessidade de ser ingerido entre as refeições para evitar diminuição da absorção e, ainda, a baixa biodisponibilidade própria do composto.

A necessidade de busca de alternativas para o controle de anemia que não exclusivamente aquelas intervenções já implantadas no Brasil, com destaque para a fortificação das farinhas de trigo e de milho enriquecidas com ferro, ficou 
Quadro 3. Prevalência de anemia em crianças (6-59 meses), em mulheres (15-49 anos). PNDS, 2009

\begin{tabular}{ccccc}
\multicolumn{5}{c}{ em mulheres (15-49 anos). PNDS, 2009} \\
\hline Região & $\begin{array}{c}\text { Crianças } \\
\text { Crianças \% }\end{array}$ & $\begin{array}{c}\text { Mulheres } \\
{[\mathrm{Hb}]<12,0 \mathrm{~g} / \mathrm{dL}}\end{array}$ & $\begin{array}{c}\text { Mulheres } \% \\
{[\mathrm{Hb}]<11,0 \mathrm{~g} / \mathrm{dL}}\end{array}$ \\
\hline Brasil & 3455 & 20,9 & 5669 & 29,4 \\
Norte & 832 & 10,4 & 980 & 19,3 \\
Nordeste & 665 & 25,5 & 1168 & 39,1 \\
Sudeste & 680 & 22,6 & 12004 & 28,5 \\
Sul & 605 & 21,5 & 1169 & 24,8 \\
Centro-Oeste & 673 & 11,0 & 1158 & 20,1 \\
\hline
\end{tabular}

Fonte: IBGE, PNDS,2009

bem visível no dia 23 de março de $2009^{29}$ quando foram divulgados os dados de um primeiro estudo nacional relativo à prevalência de anemia do projeto PNDS, 2006 (Pesquisa Nacional de Demografia e Saúde da Criança e da Mulher). Valores de prevalência de anemia entre mulheres em idade reprodutiva (15-49 anos) e crianças de 6 a 59 meses de idade estão apresentados no Quadro 3.

De forma diferente do que ocorre com a desnutrição proteico-energética, com as doenças diarreicas e com as parasitoses intestinais, a anemia alcança níveis significantes entre famílias de melhor nível socioeconômico. Essa disseminação da deficiência de ferro entre todos os estratos socioeconômicos aponta para a necessidade de estudos mais refinados sobre sua epidemiologia. Parece, pois indispensável conhecer a exata determinação que a condição socioeconômica exerce sobre o aporte de ferro na dieta e os mecanismos que relacionam este aporte às reservas orgânicas do mineral e, em sequência, à concentração da hemoglobina no sangue.

Ao mesmo tempo, a elevada prevalência de anemia entre mulheres cuja alimentação é similar à da família, descrita no PNDS, 2006, pelo menos dois anos após a implantação da fortificação das farinhas de trigo e de milho em todo o Brasil e a elevada prevalência de anemia entre crianças com acesso à suplementação de ferro enquanto lactentes ressalta a necessidade de uma discussão mais ampla abarcando também a respeito das intervenções com vistas à obtenção de resultados mais próximos das metas previstas para elas e/ ou de alternativas que, em mais curto prazo, permitam o controle dessa desnutrição. Com esse propósito, o Ministério da Saúde criou a Comissão Interinstitucional para Implementação, Acompanhamento e Monitoramento das Ações de Fortificação de Farinhas de Trigo, Farinhas de Milho e seus Subprodutos ${ }^{30}$ cuja primeira reunião foi realizada em 11 de novembro de 2009.

Como destaca Batista Fo et al., ${ }^{31}$ ainda existem diversas lacunas cujas respostas poderão levar ao controle do problema da anemia nutricional que, apesar do seu impacto epidemiológico e clínico, ainda conserva incertezas no que se refere a causas, epidemiologia diagnóstico e tratamento.

\begin{abstract}
Iron deficiency anemia is a vast epidemiologic problem evidenced by health public spending, the social consequences of increased risk in pregnancy, low production, and also by long term consequences of cognitive development. Some points in time highlight the involvement of the Brazilian government: 1977 - 1st Health Minister Meeting (INAN), with international and national specialists to discuss perspectives and interventions to control anemia; 1982/83 - distribution of iron supplement to Pregnancy Programs and 1st consultation to measure hemoglobin concentration; 1992 - Brazilian government commitment to reduce the prevalence of anemia in pregnant women by 1/3; 1994 Implementation of the "Vivaleite" Program to provide iron fortified milk to families with under 6-year-old children and incomes of up to 2 minimum wages; June 2002-2004 - wheat and corn flour fortification with iron; 2005 - Iron supplementation program to breastfeeding women; March 2009 - Reports published on the prevalence of anemia in women (15 to 49 years old) and children (6 to 59 months) in Brazil. August 2009-the 1st Inter-institutional Commission Meeting for the Implementation, accompaniment and monitoring of fortification policies of wheat and corn flour and their "subproducts" was established by the Health Minister. Rev. Bras. Hematol. Hemoter. 2010;32(Supl.2):2-7.
\end{abstract}

Key words: Public policies; iron deficiency; anemia; nutrition, Public Health.

\section{Referências Bibliográficas}

1. Batista Filho M, Rissin A. Deficiências Nutricionais: Ações Específicas do Setor Saúde para o seu Controle. Cad. Saúde Pública. 1993;9(2):130-5.

2. Enriching L Overcoming vitamin and mineral malnutrition in developing countries. Washington DC World Bank, 1994.

3. Horton S, Ross J. The economics of iron deficiency. Food Policy. 2003;28(1):51-75.

4. Gandra YR. Anemia nutricional no Brasil. Brasília, DF: Ministério da Saúde, 1977. Instituto Nacional de Alimentação e Nutrição.

5. (INAN) Relatório final: seminário sobre anemias nutricionais. Brasília, MS-INAN, 1977.99p [D.T. n 21/77].

6. Ministério da Saúde: Programa de Assistência Integral à Saúde da Mulher. Brasília: INAN, 1982.

7. Szarfarc SC A anemia nutricional ente gestantes atendidas em centros de saúde do Estado de São Paulo (Brasil). Rev. Saúde Pública., $1985 ; 19: 450-7$

8. World Health Organization Nutritional anemias. Geneva, WHO. 1968 Tech. Report Series, 405.

9. CDC (Centers for Diseases Control and Prevention). Current trends CDC criteria for anemia in children and childbearing-age women. Mor Mortal Wkly Rep. 1989;38:400-4.

10. World Health Organization Iron deficiency anaemia. Assesment, prevention and control: a guide for programme managers. Geneva, 2001 .

11. Grantham-McGregor S, Ani C. A review of studies on the effect of iron deficiency on cognitive development in children. J Nutr. 2001;131(2S-2):649S-666S.

12. Granthan-MacGregor S. Does iron-deficiency anemia affect child development? Pediatrics. 2003;112(4):948. 
13. Batista Filho M, Diniz AS. Combate às deficiências de micronutrientes no Brasil (Relatório final do Seminário sobre combate às carências de micronutrientes). Revista do IMIP, Recife, 1993; $7: 121-5$.

14. Brunken GS, Sarfarc SC. Anemia ferropriva em pré-escolares: conseqüências, formas de controle e histórico das políticas nacionais de redução da deficiência. Cad. Nutr., São Paulo, 1999;17:1-19.

15. Fisberg M, Braga JAP, et al Anemia ferropriva em pré-escolares de capitais brasileiras: resultados parciais. In: Reunión de La Sociedad Latinoamericana de Investigación Pediátrica, 38, Reunión de La Sociedad Latinoamericana de Endocrinología Pediátrica, 14, Tierra del Fuego, 2000. Resumos. Tierra del Fuego, 2000. p. 50.

16. Torres MAA, et al. Anemia em crianças menores de dois anos atendidas nas unidades básicas de saúde no Estado de São Paulo, Brasil. Rev. Saúde Pública, São Paulo, 1994;28:290-4.

17. Szarfarc SC, et al. A concentração de hemoglobina no primeiro ano de vida da clientela de serviços públicos de saúde do Brasil. Cad. Saúde Pública, Rio de Janeiro, 2004;20(1):266-74.

18. Torres MAA. Programa de fortificação do leite pasteurizado e de atividades de educação em saúde e nutrição, na prevenção e recuperação da anemia carencial ferropriva, em crianças menores de dois anos. São Paulo, 2000. Tese de Doutorado. Centro de Referência da Saúde da Mulher e de Nutrição, Alimentação e Desenvolvimento Infantil - Secretaria da Saúde do Estado de São Paulo.

19. São Paulo. 1999. Decreto no 44569 de 22/12/1999, normalizando a distribuição de leite fortificado com ferro bis glicina quelato a familias do estado de São Paulo com filhos menores de 7 anos e renda inferior a 2 salários minimos. Diário Oficial do Estado de 22/12/1999.

20. Viski MM, Augusto RA, Szarfarc SC. O projeto Vivaleite no município de Santo André. Nutrire Rev. Soc. Bras. Aliment. Nutr. 2006; 31(3):15-26.

21. Sá ACE, Szarfarc SC. Prevalência de anemia em crianças, antes e durante a participação em programa de fortificação alimentar com ferro. Nutrire Rev. Soc. Bras. Aliment. Nutr. 2009;34(2):115126.

22. Fujimori E, Szarfarc SC. Avaliação do impacto da fortificação das farinhas de trigo e de milho com ferro. Seminário. São Paulo, março, 2008.

23. Assunção MCF, Santos IS, Barros AJD, Gigante DP, Victora CG. Anemia em menores de seis anos: estudo de base populacional em Pelotas, RS. Rev Saúde Pública. 2007;41(3):328-35.

24. Queiroz AL, Szarfarc SC, Marchioni DML. A fortificação das farinhas de trigo e de milho no fornecimento de ferro para a merenda escolar. Nutrire Rev. Soc. Bras. Aliment. Nutr. 2008;33(2):63-73.

25. Costa CA, Machado EHS, Colli C, Latorre WC, Szarfarc SC. Anemia em pré-escolares atendidos em creches de São Paulo (SP): perspectivas decorrentes da fortificação das farinhas de trigo e de milho. Nutrire Rev. Soc. Bras. Aliment. Nutr. 2009;34(1):59-74.

26. Monteiro CA, Szarfarc SC, Brunken GS, Gross R, Conde WL. Long-term preventive mass prescription of weekly doses of iron sulfate may be highly effective to reduce endemic child anemia. Food Nutr. Bull., Tokyo, 2001; 22(1):53-60.

27. Brasil. Ministério da Saúde. Secretaria de Políticas de Saúde. Projeto para o controle da anemia ferropriva em crianças menores de 2 anos nos municípios do Projeto de Redução da Mortalidade na Infância. Brasília: Ministério da Saúde, 1998. 6p. [Apostila].

28. Brasil. Ministério da Saúde. Programa Nacional de Suplementação de Ferro. Brasília, 2003. [Apostila].

29. Brasil. Ministério da Saúde. Centro Brasileiro de Análise e Planejamento. Pesquisa Nacional de Demografia e Saúde da Criança e da
Mulher. PNDS, 2006. Série G Estatística e Informação em Saúde. Brasília. DF, 2009.

30. Brasil. Ministério da Saúde. Portaria no 1793/GM/agosto/2009 instituindo a Comissão Interinstitucional para implementação, acompanhamento e monitorização das ações de fortificação das farinhas de trigo e milho e seus subprodutos.

31. Batista Filho M, Souza AI, Bresani CC. Anemia como problema de saúde pública. Ciênc. saúde coletiva. 2008;13(6):1917-22.

O tema foi sugerido e avaliado pelo coeditor deste fascículo educativo, Rodolfo Delfini Cançado, e pelo board interno da RBHH, e publicado após a concordância do editor, Milton Artur Ruiz.

Conflito de interesse: sem conflito de interesse

Recebido: $12 / 01 / 2010$

Aceito: $16 / 01 / 2010$ 